\title{
O Princípio da Precaução no uso de indicadores de riscos químicos ambientais em saúde do trabalhador
}

\author{
The Principle of Precaution in the use \\ indicators of environmental chemical risks \\ to occupational health
}

Lia Giraldo da Silva Augusto 1

Carlos Machado de Freitas 2

\footnotetext{
1 Pesquisador-Adjunto do Núcleo de Estudos em Saúde Coletiva, Centro de Pesquisa Aggeu Magalhães, Fundação Oswaldo Cruz.

2 Pesquisador-Adjunto do Centro de Estudos da Saúde do Trabalhador e Ecologia Humana, Escola Nacional de Saúde Pública, Fundação Oswaldo Cruz.
}

\begin{abstract}
Taking the Principle of Precaution as a reference, this article reviews critically the intensive use of isolated biological and environmental indicators to assess chemical exposition. Vigilance and monitoring occupational chemical risk relies mainly on analytical laboratory techniques. The need to develop interdisciplinary and participative approaches is felt by the authors, who propose the surpass of traditional monitoring health practices, and it's trend to subordinate the complex to the simple. According to the Principle of Precaution, biological as well as environmental indicators of exposure can only achieve their true value when applied within a global view of the system under analysis. This demands a comprehensive change of perspective in our scientific and social practices of chemical risk's control and prevention.

Key words Health Monitoring; Occupational Exposition; Chemical Risk; Principle of Precaution; Interdisciplinarity
\end{abstract}

Resumo Tendo como referência o Princípio da Precaução, o presente artigo faz uma abordagem crítica do uso isolado de indicadores biológicos e ambientais no monitoramento e vigilância em saúde frente ao risco de exposição ocupacional às substâncias químicas. Apontamos para a necessidade da abordagem interdisciplinar e participativa. Propomos uma reflexão na perspectiva da superação das tradicionais práticas em saúde do trabalhador, que subordinam o complexo ao simples. Do ponto de vista do princípio precaucionário, os indicadores biológicos de exposição e efeito, bem como os de contaminação ambiental, só possuem valor quando integrados à globalidade do sistema em estudo, obrigando-nos a repensar as práticas científicas e sociais tradicionais de controle e prevenção de riscos de origem química.

Palavras-chave Vigilância em Saúde; Exposição Ocupacional; Risco Químico; Princípio da Precaução; Interdisciplinaridade 
Quotidianamente defrontamo-nos com notícias referentes aos riscos que processos industriais ou produtos químicos podem causar a nossa saúde e ao meio ambiente. O paradoxo é que, por um lado, se os avanços científicos e tecnológicos contribuíram para a redução da prevalência de determinadas doenças associadas à fome e às pestilências, por outro, eles fizeram surgir novos riscos, como os de origem química, cujo aumento contribui para o crescimento das concentrações dessas substâncias - normalmente inexistentes em ambientes não industrializados -, a elas expondo todos os dias milhões de pessoas nos seus locais de habitação ou trabalho, na cadeia alimentar, no solo em que pisam, no ar que respiram e nas águas que consomem (Freitas e Gomez, 1997; Porto e Freitas, 1997).

Mesmo que consideremos apenas a dimensão biológica, podemos afirmar que a saúde humana das populações expostas a estas substâncias químicas está sujeita a uma multiplicidade de possíveis efeitos, tais como a teratogênese, a carcinogênese, a mutagênese e danos a órgãos-alvo específicos.

Atualmente, cerca de 70 mil destas substâncias são utilizadas no quotidiano pelo homem, das quais perto de 40 mil, em significativas quantidades comerciais (IPCS and IRPTC, 1992). Calcula-se que aproximadamente 6 mil substâncias apenas tenham sido avaliadas como minimamente adequadas no que diz respeito aos riscos que oferecem ao homem e ao meio ambiente, ou seja, tenham passado por estimativa que inclua não somente testes de toxicidade aguda, mas também subcrônica e crônica, testes eco-toxicológicos e estudos epidemiológicos convenientes após seu emprego em larga escala social. A este quadro, acrescente-se a capacidade de inovação tecnológica no ramo químico, que não só vem tornando mais complexos os sistemas tecnológicos de produção, como vem colocando disponíveis no mercado, a cada ano, entre mil e 2 mil novas substâncias (Porto e Freitas, 1997).

Foi em resposta à crise ambiental criada por tecnologias como a de processos industriais e produtos químicos que se desenvolveram os métodos científicos de avaliação de riscos, refletindo a tendência para prever, planejar e alertar quanto aos riscos - em vez de dar respostas ad hoc às crises que elas geraram -, bem como a idéia de que as decisões regulamentadoras sobre os mesmos seriam politicamente menos controversas se pudessem ser tecnicamente mais rigorosas e fundadas em firme base 'factual', como, por exemplo, o desenvolvimento e o emprego de indicadores biológicos de exposição. Essa base deveria ser construída a partir dos dados disponíveis de exposição às substâncias químicas, além de suplementados por cálculos, extrapolações teóricas e julgamentos 'objetivos', oriundos de análises estatísticas, de modo a obter-se um valor esperado, o qual seria utilizado para processos decisórios que envolvessem a utilização de tecnologias consideradas perigosas em larga escala social e seu controle (Starr et al., 1976; Otway, 1985; Renn, 1985 e 1992).

Neste contexto é que se desenvolve a avaliação dos riscos à saúde e ao meio ambiente, a qual, para o caso das substâncias químicas, tem como seus pilares, no campo da saúde, a Toxicologia (experimentos em animais de laboratório) e a Epidemiologia (populações expostas aos agentes perigosos comparadas com as não expostas), que procuram identificar e quantificar as relações entre os potenciais agentes de riscos químicos e os danos biológicos observados nos seres vivos, humanos e não-humanos. Mediante modelagens, o agente causal é isolado das variáveis intervenientes ou confounders. O que há de comum entre essas perspectivas é o fato de reduzirem o risco à dimensão única, representando a média com base em espaços e tempos estáveis (Renn, 1992).

Entretanto, nas últimas décadas, o reconhecimento e a aceitação dos inerentes limites do conhecimento científico acerca de problemas ambientais e das incertezas que o acompanham, assim como dos perigos associados à capacidade de inovação e emprego em larga escala social de produtos e processos industriais perigosos - que é maior do que a capacidade de avaliar adequadamente seus riscos - têm provocado mudanças nas políticas ambientais, científicas e tecnológicas em direção à filosofia preventiva, fundada em princípio regulatório particular que lhe dá efeito prático: o Princípio da Precaução (Wynne, 1992).

\section{Princípio da Precaução}

De acordo com Wynne (1992), o Princípio da Precaução foi desenvolvido de início na Alemanha, como meio de justificar a intervenção regulamentadora para a restrição das descar- 
gas de poluição marinha na ausência de provas consensuais quanto aos danos ambientais. Este princípio tem sido tomado como referência em outras áreas - incluindo as mudanças climáticas globais - e caracteriza-se por requerer que as decisões acerca de processos industriais e produtos perigosos sejam deslocadas da ponta final do processo - ou seja, quando uma inovação tecnológica já é empregada em larga escala social - para a ponta inicial do processo - ou seja, quando uma inovação tecnológica ainda se encontra em teste para avaliar seu desempenho em termos de eficácia e segurança.

A aplicação do Princípio da Precaução envolve não só o reconhecimento e a exposição das inerentes incertezas no que diz respeito aos eventuais efeitos das substâncias químicas sobre os seres humanos e o meio ambiente, mas também a admissão de nossa ignorância em relação ao problema e à indeterminância. A complexidade e uma série de limites e incertezas quanto às avaliações técnicas de riscos ampliam-se quando levamos em conta que os processos saúde-doença ligados à exposição a substâncias químicas envolvem interações não-linerares de aspectos biológicos, psicológicos e sociais que são altamente acoplados, possibilitando múltiplas e inesperadas interações, as quais se tornam, muitas vezes, incompreensíveis e invisíveis aos seres humanos a curto prazo. Junto a isto temos ainda que considerar o número e a diversidade de espécies de seres vivos que, por vezes, apresentam grande variabilidade genética, além das diferenças entre as composições químicas de solos, águas e atmosferas em ambientes específicos, que contribuem para aumentar a complexidade do problema e as incertezas a seu respeito. Nesta perspectiva, as limitações do conhecimento científico disponível, no que concerne aos riscos, são potencialmente mais sérias, porque, como observa Wynne (1992), os sistemas em questão, não sendo artefatos tecnológicos, não podem ser projetados, manipulados e reduzidos dentro dos limites do conhecimento analítico existente, obrigando-nos a ir além dos conceitos de risco e incertezas.

O conceito de risco faz supor que o comportamento do sistema em questão é basicamente bem conhecido e que as chances de diferentes resultados podem ser definidas e quantificadas por meio de análises estruturadas de mecanismos e probabilidades. As incertezas - que lhe são associadas e estão na sua origem - surgem quando conhecemos os importantes parâmetros do sistema, porém não a distribuição de probabilidades, existindo métodos sofisticados para estimá-las e aos efeitos de seus resultados. Nas incertezas conhecemos o que ainda não conhecemos. A ignorância, por definição, refere-se ao que escapa ao reconhecimento, sendo ela endêmica ao conhecimento científico, o qual tem de reduzir a estrutura do saber ao que é mais adequado para os métodos e modelos de análise. $\mathrm{Na}$ indeterminância, a cadeia causal de conhecimento é aberta não só por conta das interações não-lineares de aspectos que são altamente acoplados e não se restringem somente à dimensão biológica, mas também pela variabilidade genética dos seres vivos e pela diversidade social e ambiental dos contextos em que as exposições químicas ocorrem (Wynne, 1992).

Assim, o reconhecimento e adoção do Princípio da Precaução não só tem como conseqüência mudar a responsabilidade das provas científicas para o gerador de riscos, passando-se a exigir que este desenvolva estudos completos antes do emprego de uma substância. Deste modo tenta-se evitar que, como na maioria dos casos, venha a constituir mais um experimento em larga escala social em tempo real. Tem implicações também na própria estrutura do conhecimento científico adotado nas avaliações de risco, convertendo-se em um dos pontos de referência para mudança paradigmática na ciência e nos processos decisórios que envolvem riscos.

Assim, para os casos de substâncias em que seja razoável antecipar que podem ocasionar danos irreversíveis à saúde e ao ambiente e que há ausência de provas científicas suficientes quanto aos danos potenciais, ao invés de continuar produzindo e manipulando o produto até que se prove que ele é danoso, como ocorre na atualidade, a aplicação do Princípio da Precaução coloca a necessidade de parar a produção e o manuseio até que se desenvolvam conhecimentos suficientes sobre a inoquidade do produto.

\section{Indicadores biológicos de riscos químicos ambientais}

Os indicadores biológicos de exposições ambientais aos riscos de origem química nos locais de trabalho, que servem de base 'factual' para as avaliações de risco e vigilância em saú- 
de, compõem elementos privilegiados para a discussão do Princípio da Precaução. Ademais, o debate crítico do uso destes indicadores reveste-se de importância fundamental, uma vez que constituem os parâmetros básicos - os limites de exposição ocupacional - da legislação para controle e prevenção dos riscos associados às substâncias químicas.

Os indicadores biológicos de exposição são o resultado da estimativa da inalação, ingestão ou dermo-absorção de compostos químicos presentes no meio ambiente, muitos dos quais suspeitos de propriedades teratogênicas, mutagênicas e carcinógenas para humanos (Howe et al., 1986). A significância de avaliações individuais e coletivas desses indicadores está na possibilidade de identificar aqueles que possam servir ao monitoramento de danos precoces, principalmente em nível celular. Nesse sentido, a identificação de indicadores de exposição é importante auxiliar da Epidemiologia em programas de vigilância à saúde.

Para alguns tipos de efeitos biológicos possíveis, pode-se ter um ou mais indicadores de efeito específico. A média da concentração biológica de uma substância química em fluidos orgânicos, por exemplo, pode constituir um bom indicador de exposição aguda e, portanto, de risco para a saúde. Entretanto, em relação aos efeitos crônicos - por exemplo, o câncer - os dados do monitoramento biológico são absolutamente insuficientes para serem usados como demonstrativos da exposição individual ou coletiva, atual ou pregressa, em especial para dimensionar o risco e garantir a saúde dos grupos vulneráveis (Ashby, 1988).

Além disto, deve-se levar em conta que, tradicionalmente, os indicadores construídos a partir de dados provenientes de monitoramentos ambiental e biológico de exposição e de efeito são utilizados na avaliação de risco de forma isolada e descontextualizada, sem atentar para as inter-relações e interdependências de todos os elementos que compõem o espaço socioambiental no qual se desenvolve a atividade de trabalho, constituindo isto uma das maiores limitações à estimativa de riscos para a saúde humana.

Considerando-se que as diferenças individuais e as características da exposição determinam a necessidade de abordagem integrada na avaliação de riscos e de efeitos, os indicadores biológicos - úteis ao monitoramento de efeitos adversos - deveriam ser emprega- dos de forma integrada com outros elementos de avaliação da saúde, fazendo-se acompanhar de medidas de prevenção e controle. Assim, esses indicadores deveriam ser utilizados apenas como garantia de que o ambiente se encontra sob controle e não como garantia de segurança à saúde, pois os fenômenos biológicos envolvidos nesses processos são complexos, de nível superior - isto é, hierarquicamente superiores na escala filogenética -, não se podendo subordiná-los, por exemplo, aos procedimentos elementares da química analítica. Estes, na verdade, são medidas de concentração de agentes químicos e seus metabolitos no ambiente ou nos fluidos humanos e pertencem a níveis inferiores na escala da complexidade, pois se reduzem a equações tipo: concentração $=$ massa/volume (Novaes, 1992). Semelhantes questões têm sido polêmicas, em especial, as relativas aos chamados limites de exposição ocupacional.

A toxicidade crônica de determinadas substâncias - manifestada, por exemplo, pelo câncer e hipersensibilidade - nem sempre tem relação com outras propriedades tóxicas agudas da mesma substância e que, com freqüência, são muito graves, exigindo também vigilância permanente. No entanto, as intoxicações agudas, por seu dano imediato e clinicamente evidentes, têm recebido mais atenção e obtido considerável grau de sucesso dentro da abordagem linear de dose-efeito que ganhou legitimidade.

Já as manifestações crônicas, que são mascaradas por outros fenômenos e mediados pela variável tempo e susceptibilidade individual, exigem da metodologia de análise um modelo explicativo não linear. As dificuldades metodológicas para aproximação a esses problemas é fato da atualidade que surge pela força que vem assumindo o paradigma da prevenção nas ações de vigilância em saúde. Também desenvolve-se maior consciência sanitária e ecológica dos trabalhadores de nossa época em relação aos problemas de exposição ocupacional.

Embora haja consenso da comunidade científica internacional de que não existem níveis seguros de exposição aos cancerígenos, por exemplo, uma vez que, teoricamente, uma simples mutação celular pode levar ao câncer em seres humanos (IARC, 1982), na prática impõe-se o modelo dose-efeito da bioquímica pelo tradicional monitoramento da saúde dos trabalhadores. 
Para avaliação dos efeitos crônicos, os dados biológicos-individuais e os coletivos decorrentes do monitoramento de exposição ocupacional para substâncias químicas deveriam ser coletados periodicamente em um sistema de vigilância epidemiológica. No entanto, tais informações são entendidas como pertencentes ao espaço privado das empresas, as quais, em nossa realidade, ocultam-nas ou a elas dificultam o acesso.

Idealmente, um indicador poderia ser a concentração do composto tóxico nos diversos tecidos do organismo. Mas como há restrições éticas e técnicas para sua realização in vivo, utiliza-se a determinação das concentrações do agente e de seus metabolitos no sangue, o que deve refletir sua concentração nos outros tecidos (Monster, 1988). No entanto, esta limitação à avaliação toxicológica fica na dependência de mais um mediador complexo no processo de análise. Sabemos que influências endógenas - algumas não plenamente conhecidas - são variáveis de difícil controle. Aqui, também, o modelo linear não se aplica com tanto grau de certeza, mas o raciocínio simplista impõe decisões que não correspondem à complexidade dessa realidade e que, com freqüência, acarretam novos riscos.

\section{Uma perspectiva crítica dos indicadores biológicos}

Os chamados indicadores biológicos de exposição e de efeito merecem profunda reflexão conceitual. Sua utilização só é eticamente sustentável se contar com o conhecimento daqueles que estiverem potencialmente expostos e se os efeitos monitoráveis forem de alterações precoces, que permitam o completo reparo dos danos. Caso contrário, não serão indicadores para fins de monitoramento ou de vigilância voltados à prevenção em saúde, mas, sim, exames complementares - próprios da clínica e não da saúde pública - para diagnóstico das patologias decorrentes das exposições aos agentes nocivos ambientais (Tarlau, 1990; Waldman, 1991).

Faz-se necessário apresentar a diferença conceitual entre Vigilância em Saúde e Monitoramento. Neste sentido, os conceitos propostos por Waldman (1991) parecem-nos mais próximos da abordagem sistêmica defendida neste artigo. Para esse autor, a vigilância em saúde é constituída de três subsistemas integrados:

- "Subsistema de informação para ações de controle": com atribuições de coleta e análise sistemática de dados relativos a eventos específicos adversos à saúde e/ou respectivos programas de controle para indicar as medidas imediatas de controle, com fundamento nas recomendações técnicas disponíveis, divulgando-as amplamente;

- "Subsistema de inteligência epidemiológica": incorpora os conhecimentos científicos e tecnológicos disponíveis para análise sistemática de informações, a fim de elaborar recomendações e oferecer as bases técnicas para ações de controle, divulgando-as amplamente; - "Subsistema de pesquisa": tem por atribuição desenvolver pesquisas científicas e tecnológicas voltadas à solução de problemas prioritários e/ou emergentes em saúde pública, identificados nos demais subsistemas.

O termo monitoramento ou 'monitorização’ significa procedimentos contínuos de mensuração e análise dos indicadores de saúde e de riscos ambientais integrados ao sistema de vigilância à saúde, com o objetivo de oferecer subsídios para aplicação de medidas preventivas, de controle e de avaliação (Waldman, 1991). Portanto, para a definição das linhas de ação em vigilância da exposição química devem ser consideradas as seguintes dificuldades e restrições: 1) a determinação de um risco aceitável, pois, além dos fatores capazes de precisar um estado patológico relativo tanto ao ambiente como à dimensão biológica do hospedeiro (Vesel, 1987) há os que dependem da dimensão política (Vanhoorne, 1988); e 2) a extrapolação de dados entre espécies não pode proceder por simples equação matemática relacionada ao peso corporal e à taxa metabólica específica (conceito de coeficiente de segurança), conforme alertam Tyler \& Balantyne (1988).

A Dose Letal (DL-50) exemplifica esta aplicação linear. Esta tem configurado o principal fundamento da toxicologia a sustentar práticas de monitoramento de exposição aos produtos químicos e a dar apoio à legislação em vigor, com seus Limites de Tolerância que servem mais aos interesses econômicos da produção do que à prevenção em expostos.

Outro fator que não é em geral ponderado nos estudos de efeito ou no estabelecimento de indicadores de exposição é a ação sinérgica das substâncias presentes no ambiente de 
trabalho (Veerbeek et al., 1986). A Toxicologia tem sido, portanto, uma disciplina cujo modelo linear presta-se a tais interesses. Segundo Lieber (1991) "o desenvolvimento da toxicologia sempre exigiu muito mais que experimentação, observação e reconhecimento científico... ela sempre foi uma ciência associada ao poder e, inevitavelmente, sujeita a interesses em conflito ou a segredos, por vezes de importância estratégica." Neste sentido, Doull \& Bruce (1986) concluem no que diz respeito às indústrias: "o desenvolvimento dessa disciplina está relacionada às condições sócio-políticas".

Estas citações têm por objetivo enfatizar que, para a prevenção em situações de exposição química, outro fator a ser levado em conta, além da dimensão biológica, é a complexidade dos fenômenos bio-socioambientais que possuem mais incertezas do que se suspeita e cuja desconsideração amplia a ignorância concernente aos processos saúde-doença na exposição às substâncias químicas (Wynne, 1992).

\section{Restrições ao uso dos limites de exposição ocupacional}

Outra questão ligada aos programas de vigilância à saúde diz respeito aos limites de exposição, os quais representam papel importante na questão ocupacional, do ponto de vista da efetiva proteção dos trabalhadores sujeitos ao risco.

Castleman \& Ziem (1988) vêm acusando a influência empresarial nos valores dos limites de tolerância - Threshold Limit Values - TLVs - e o uso de procedimentos irregulares e nãocientíficos pelo Comitê do TLV da American Conference of Governmental Industrial Hygienists-ACGIH. A esse respeito, denunciaram:

"Comunicações não publicadas de empresas foram importantes no desenvolvimento de TLVs para 104 substâncias. Para 15 delas, a documentação do TLV foi baseada somente em tais informações... Aos representantes de empresas, listados como consultores do comitê, desde 1970, foi dada a responsabilidade principal no desenvolvimento de TLVs para produtos químicos, de propriedade das companhias que os empregavam... Não foram feitas pesquisas bibliográficas sistemáticas na preparação das documentações sobre centenas de produtos químicos..."

Em razão desses reclamos, a ACGIH adotou um 'código de honra', no qual os membros do comitê de TLV meramente declaram, mediante termo de compromisso, que não têm conflitos de interesse (Tarlau, 1990). Essa autora propõe com convicção que "dada a limitação de nossa ciência, controles podem e devem ser colocados no local (de risco), mesmo quando as causas não são bem conhecidas ou completamente entendidas e muito menos quantificadas". Segundo Tarlau, a realidade é que, para a vasta maioria dos produtos químicos, temos poucos ou nenhum dado sobre toxicidade crônica. Mesmo quando os temos, não sabemos ao certo os efeitos químicos na função pulmonar, no sistema nervoso, no endócrino ou no imunológico, no reprodutivo ou em outras funções vitais do organismo. Sem tais dados, são falsas as afirmações de que conhecemos quais exposições são permitidas por não prejudicarem os trabalhadores.

Nos ambientes de trabalho - em especial, nos das indústrias químicas - existe a prática do relativismo do risco, isto é, ele ser ou não aceitável, e decorre de interpretação não mais vinculada aos eventos naturais, mas associada aos processos produtivos do interesse econômico (Sharlin, 1989).

O episódio da Portaria no 3, de 10/03/94, editada pelo Ministério do Trabalho, referente ao benzeno, exemplifica esse relativismo. Sua publicação deu origem a conflito com setores empresariais por revogar o Limite de Tolerância estabelecido pela Portaria 3.214/78 (NR-15, ANEXO-13) que permitia exposição de $8 \mathrm{ppm}$ (partes por milhão) para jornadas de 48 horas semanais de trabalho.

A nova Portaria foi motivada pelo reconhecimento (tardio) de que o benzeno é cancerígeno e que não se poderia mais admitir limite legal de exposição ocupacional a esse agente químico (Augusto,1991; M.T., 1993). A interpretação dada por empresários foi de que se estava definindo 'risco zero', que constitui situação ideal, sem correspondência com a realidade da vida, mostrando-se, portanto, inexeqüível. No argumento empresarial afirmou-se que, em virtude de ser impossível eliminar tal risco, ele só pode ser minimizado (C.N.I., 1994; Freitas \& Arcuri, 1996).

No entanto, a referida portaria foi elaborada após ampla revisão do tema e estava conceitualmente fundamentada. Neste sentido, podemos citar Sass (1988), para quem um risco não pode ser aceitável, em se tratando de substâncias comprovadamente tóxicas, e o objetivo deverá ser sempre o de reduzir a exposi- 
ção a zero. Desta forma, até é possível definirse limite de tolerância para avaliar as medidas que objetivem o controle do risco e sua eliminação, mas ele não deve ser empregado para garantir segurança à saúde e nem para descaracterizar os efeitos nocivos decorrentes da contaminação ambiental nas situações de concentração abaixo do limite estabelecido.

Os efeitos decorrentes das exposições químicas só podem ser definidos, em geral, dentro das limitações dos instrumentos adotados e sob limitado número de expostos. Neste sentido, o conceito de exposição segura não pode ser considerado positivo, isto é, o valor aceitável para um grupo não pode ser extrapolado com certeza para outros (Castleman \& Ziem,1988). Semelhante afirmação contrasta com a prática de monitoramento de risco que, com freqüência, busca firmar a segurança para aquilo que, na verdade, tem alto grau de incerteza. Esta tem sido fonte de conflito para a maioria das situações que enfrentamos na vigilância em saúde dos trabalhadores.

Quando se trata de risco, outra questão de discórdia é a de sua percepção, a qual também é relativa e depende de fatores sociais. Neste sentido, é freqüente o conflito decorrente da chamada evidência científica versus a percepção popular. Cada vez mais, a solução para estas questões tem sido a negociação entre as partes interessadas. Parece-nos que esta dimensão social deva ser incorporada como saber legítimo, sem a contraposição do saber científico como o único capaz de dar respostas aos problemas enfrentados na exposição química. Na perspectiva do Princípio da Precaução, é na fronteira destes saberes que estão os espaços para a construção da interdisciplinaridade e de processos decisórios mais amplos em seu escopo e impacto (Wynne, 1992).

\section{Contribuição da epidemiologia na avaliação de exposição química}

Os estudos epidemiológicos são considerados as melhores fontes de informação a respeito dos efeitos de exposição a agentes nocivos: "uma vez que estão baseados nas condições da realidade a que estão expostos os seres humanos" (Wunsch, 1992). Nesta orientação, os marcadores biológicos de exposição e efeito são auxiliares preciosos da Epidemiologia, que pode assim trabalhar com efeitos mais preco- ces e permitir real intervenção preventiva (Ashby, 1988).

Para Thomas (1979), a Epidemiologia tem limitações quanto ao estabelecimento do risco determinado por novas substâncias ou novas condições de trabalho. Isto ocorre não tanto pelo método, mas porque as informações ficam na dependência das empresas e dos profissionais de saúde - muitas vezes, por elas contratados -, sujeitos aos conflitos de interesse e à interferência de condições particulares, observadas nos expostos ou nos locais de trabalho, que restringem sua aplicação. Por sua vez a Organização Mundial da Saúde (OMS, 1977, 1980 e 1981) enfatiza que as exposições múltiplas a diversos agentes nocivos no ambiente de trabalho são fatores intervenientes e restritivos dos estudos epidemiológicos, bem como os fatores individuais, como, por exemplo, a suscetibilidade individual e condições de vida. Entende-se como susceptibilidades individuais, as variações biológicas em virtude de gravidez, variações hormonais, diferenças genéticas, sexo, idade, raça, nutrição e doenças prévias ou atuais.

O conceito de risco e as variáveis que adotamos para seu estudo são importantes para as ações de prevenção em situações de exposições químicas. Classicamente, define-se risco como a probabilidade de ocorrência de um evento. Aqui residem outras dificuldades metodológicas dos estudos epidemiológicos concernentes a especificidades das populações (MacClean, 1979) e que surgem tanto no momento da definição do nível de dano quanto ao fato de se lidar com grupos de expostos muito pequenos.

Mais recentemente, no entanto, métodos específicos têm sido desenvolvidos na Epidemiologia para análise de exposições múltiplas (Kundiev \& Navakatikyan, 1986). Esperamos que a força explicativa da Epidemiologia seja ampliada, uma vez que entendemos ser instrumento fundamental para a compreensão dos processos saúde-doença nas coletividades. Nesse caminho, duas correntes dentro da Epidemiologia destacam-se no sentido de dar conta dessa complexidade: 1) Susser (1985) apresenta, como ponto de partida de todo o estudo da causalidade, um método que desemboca na necessária e constante diferenciação do objeto de estudo (ou ação) em variáveis (Duval, 1995). Consciente da variabilidade dos fenômenos biológicos e sociais, Susser (1985) recomenda cinco estratégias para elaborar in- 
ferências causais relativas a eles: "as características do recorte que se faz da realidade, a depuração dos modelos causais hipotetizados, a análise das associações observadas, o uso do cálculo de probabilidade para estimar sua significação e os critérios do investigador". 2) Breilh et al. (1990) ponderam as evidências em saúde como conjunto integrado de processos e assinalam que não se pode assumir cada uma das variáveis e indicadores do estudo ou avaliação como entidade isolada, senão como processo concatenado e que forma a unidade global a ser considerada.

As questões que temos enfrentado na prática são: como fazer a vigilância em saúde do trabalhador nas situações de exposição aos agentes nocivos nos ambientes de trabalho em particular, os referentes aos químicos - e como a prevenção de danos à saúde poderá ser auxiliada pelo monitoramento ambiental e biológico?

\section{Enfoque sistêmico}

Tem crescido a fundamentação filosófica de enfoque 'holístico' para fixação de limites de exposição. Zielhuis \& Wibowo (1989) listaram dez princípios gerais para essa nova abordagem (Quadro 1)

Analisando esse quadro, assinalamos certos princípios que julgamos relevantes para a vigilância da exposição química nos processos produtivos: 1) a falácia da crença no homem médio. Na realidade, há necessidade das considerações relativistas das condições socioeconômicas, sanitárias, culturais e circunstanciais na análise da exposição dos trabalhadores a produtos químicos; 2) a insuficiência científica para dar conta de todos os elementos desse sistema; 3 ) a necessidade de participação política; e 4) a superação da racionalidade instrumental das análises ambientais, na qual as decisões não podem ser restritas aos dados quantitativos.

A proposta desses autores é ressaltar o aspecto ético na distribuição de benefícios e de riscos, em particular, o direito à informação e à eqüidade.

Entre os aspectos éticos, Lieber (1991) comenta que "a garantia de estar plenamente informado e de participar daquilo que lhe diz respeito é um direito universal consagrado ao homem. O estabelecimento de limites de exposição deve deixar claras as diferenças entre o que cien- tificamente é aceitável e o que de fato deve ser aceito".

Entre as questões econômicas que estão na pauta do enfoque holístico acerca dos custos, pergunta-se: quem paga a conta do descontrole ambiental? Em Saúde do Trabalhador, observamos que aqueles que ocupam funções hierárquicas inferiores assumem maior risco. E estes se sobrepõem ao fato de possuírem piores condições de vida e maior dependência da empresa (menor liberdade). Portanto, quem tem pago a conta são os próprios trabalhadores e a sociedade em geral, que acabam demandando maior investimento público para corrigir os danos provocados ao sistema socioambiental.

As questões ético-econômicas envolvidas no problema do risco ambiental vão ainda mais longe. Quando estamos tratando da saúde da mulher trabalhadora, por exemplo, cabe questionar: por que se deve aplicar à mulher o mesmo limite de exposição? Iguais direitos ao trabalho não implicam equivalência de riscos à saúde, principalmente quando estes riscos são necessariamente diferentes, em relação à própria diferença biológica. Além disto, ao feto e ao lactente cabem os seus próprios direitos, independentemente daqueles devidos ao pai ou à mãe trabalhadora (Lieber, 1991).

\section{Considerações finais}

Existe um custo social e ambiental decorrente do modelo simplista aqui questionado e criticado e que não é assumido pelos setores onde os riscos são gerados. Aprofundar a discussão da eficácia desse modelo de monitoramento - limitado aos métodos analíticos quantitativos e fetichizados pela parafernália instrumental - é fundamental para os trabalhadores expostos, no sentido de definição da política de prevenção dos danos à saúde e ao meio ambiente. Repensar o modelo não depende de "novo tipo de teoria", como afirma Stengers (1990), mas de "nova visão de mundo", que se constrói no compromisso ético com a vida, pois o conhecimento científico, no qual construímos os riscos e os sistemas ambientais, é também atravessado por julgamentos sociais tácitos sobre o próprio mundo em que vivemos. Como observa Wynne (1992), a ausência de reconhecimento disto distorce o debate público e o entendimento das próprias relações entre o conhecimento de especialistas, 
Quadro 1

Princípios gerais no estabelecimento de limites de exposição ocupacional.

\begin{tabular}{ll}
\hline Princípio & Comentário \\
\hline 1) Critério ergonômico e higiênico & $\begin{array}{l}\text { Minimizar a presença de xenobióticos que não contribuem } \\
\text { para a saúde humana. }\end{array}$ \\
\hline 2) Saúde humana e ecologia & $\begin{array}{l}\text { Minimizar a presença de xenobióticos que põem em risco } \\
\text { a sobrevivência da espécie. }\end{array}$ \\
\hline 3) Variações intra e interpessoal & $\begin{array}{l}\text { Não existe homem médio; proteger a maioria não tem } \\
\text { na exposição e resposta }\end{array}$ \\
& significado se não se estabelecem as circunstâncias; o fator \\
& O risco assumido deve estar claramente explícito. \\
\hline
\end{tabular}

4) Saúde e danos à saúde - O padrão estabelecido deve prevenir danos à saúde; a resposta humana aos vários fatores internos é limitada a um número de expressões. Estas nem sempre podem ser automaticamente relacionadas à exposição, sem considerar outros fatores de vida.

- Critérios baseados em saúde ou efeito adverso são relativos no tempo e entre as nações, pelas suas condições próprias.

\begin{tabular}{|c|c|}
\hline 5) Desconforto & $\begin{array}{l}\text { - Efeito subjetivo também é efeito; o trabalhador é um indivíduo } \\
\text { e reage como um todo. } \\
\text { - Prevenir desconforto é manter saúde. }\end{array}$ \\
\hline 6) Exposição e dose & $\begin{array}{l}\text { • Exposição externa não pode ser genericamente relacionada } \\
\text { à exposição interna (dose interna) pela individualidade nos } \\
\text { fatores cinéticos e pelas particularidades da ação dinâmica. } \\
\text { - A média ponderada de concentração pode ser indicador de } \\
\text { exposição, mas não de risco à saúde. } \\
\text { - As variações cronobiológicas determinam diferenças cinéticas } \\
\text { e dinâmicas. } \\
\text { - Limites de exposição se aplicam ao controle da exposição } \\
\text { externa, mas, em geral, não do risco à saúde, se estiverem } \\
\text { expressos em média ponderada. }\end{array}$ \\
\hline $\begin{array}{l}\text { 7) Limite biológico } \\
\text { de exposição (LBE) }\end{array}$ & $\begin{array}{l}\text { - Desestimulariam o controle do ambiente, conflitos éticos } \\
\text { na preservação dos direitos individuais. } \\
\text { - LBSs devem ser aplicados como forma complementar e rotineira } \\
\text { estimulados na fixação de novos valores. }\end{array}$ \\
\hline $\begin{array}{l}\text { 8) Extrapolação de animais para } \\
\text { os homens }\end{array}$ & $\begin{array}{l}\text { - Modelos halométricos como alternativa ao coeficiente fixo } \\
\text { tradicional de segurança (100 vezes) } \\
\text { - Considerar aspectos toxicocinéticos e toxicodinâmicos } \\
\text { distintos entre espécies. }\end{array}$ \\
\hline $\begin{array}{l}\text { 9) Estimativas, opções, decisões } \\
\text { e "valores ao homem" }\end{array}$ & $\begin{array}{l}\text { - Balanço de risco e benefícios, mas benefícios a quem: } \\
\text { trabalhador, empresa ou sociedade? } \\
\text { - Conflito de diferentes interesses, em diversos países; decisão } \\
\text { dos homens para os homens não pode restringir-se a números. } \\
\text { - Chances e decisões devem ser explicitamente discutidas. }\end{array}$ \\
\hline 10) Medo do risco e risco do medo & $\begin{array}{l}\text { - Ênfase da mídia, limitação e inadequação do medo, } \\
\text { conhecimento, conflito entre a expectativa absoluta e a } \\
\text { realidade relativa na ocorrência de doença (o conceito } \\
\text { probabilista de risco). } \\
\text { - O risco ao trabalhador pode, em geral, ser pequeno, mas não } \\
\text { se aceita viver com risco imposto por outros. } \\
\text { - A informação do risco deve ser clara e profissional. }\end{array}$ \\
\hline
\end{tabular}

Zielhius \& Wibowo (1989) apud Lieber, 1991. 
bem como a escolha de valores públicos na construção dos processos decisórios que regulamentam tecnologias ambientalmente sustentáveis, limitando o escopo da concepção de mudanças sociais e ambientais. Nesta perspectiva, a adoção do Princípio da Precaução pode desempenhar papel importante ao reorientar tanto o conhecimento científico como os processos decisórios que envolvem riscos químicos.

Parcela significativa dos trabalhadores expõe-se, na atividade de trabalho, a uma combinação de cargas ambientais e sociais. $\mathrm{O}$ desgaste que estas impõem a sua saúde não pode ser captado por indicador único, uma vez que fenômenos muito complexos estão envolvidos nesse sistema (Laurell \& Noriega, 1989). Des- ta forma, as questões que envolvem a vigilância em saúde dos trabalhadores - em particular, nas situações de risco de exposição a produtos químicos - exigem abordagem coerente com a compreensão da realidade como sistema aberto em que todos os seus elementos são interdependentes, interdefiníveis, e cujas relações não são lineares (Garcia, 1986). A contribuição disciplinar seja da Epidemiologia, da Clínica, da Toxicologia ou de qualquer outro âmbito não pode ficar descolada da globalidade do problema. As dimensões bio-éticosociais que, em geral, estão anuladas no modelo simplista de monitoramento de risco são incorporadas na abordagem sistêmica, resultando disto maior eficácia nas ações de prevenção.

\section{Referências}

American Conference of Governmental Industrial Hygienists (ACGIH) 1991. Threshold Limit Values for Chemical Substances and Physical Agents and Biological Exposure Indices. ACGIH, Cincinati.

Ashby J 1988. Comparison of techiniques for monitoring human exposure to genotoxic chemicals. $\mathrm{Mu}$ tation Research Res 204: 543-551.

Augusto LGS 1991. Estudo Longitudinal e Morfológico (Medula Óssea) em Pacientes com Neutropenia Secundária à Exposição Ocupacional e Crônica ao Benzeno. Dissertação de Mestrado. Faculdade de Ciências Médicas, Unicamp. Campinas.

Breihl J, Granda E, Campara A, Yépez J, Paéz R \& Costales P 1990. Deterioro de la Vida: un Instrumento para el Analisis de Prioridades Regionales en lo Social y la Salud. Centro de Estudios y Assessoria en Salud. Corporación Editora Nacional, Serie Biblioteca de Ciencias Social, v. 28. Quito.

Castleman BI \& Ziem GE 1988. Corporate influence on threshold limit values. American Journal of Industrial Medicine 13: 531-559.

C.N.I. - Confederação Nacional das Indústrias 1994. Relatório Técnico. Ref. Portaria n. 3 de 10/3/94, do Ministério do Trabalho, mimeo.

Doull J \& Bruce MC 1986. Origen and scop of toxicology. In CD Klaassen, MO Amdur \& J Doull (eds) Casarett and Doull's Toxicology. The Basic Science of Poisons. MacMillan Publi. Co., New York.

Duval GB 1996. Metodo de Reconstruccion de los Niveles Nutricionales de una Población: Fundamentación Sistemica. Tese de Doutorado. Centro de Investigación y de Estudios Avanzados. Instituto Politecnico Na- cional - Sección de Metodologia y Teoria de la Ciencia. México, mimeo.

Freitas CM \& Gomez CM 1997. Análise de riscos tecnológicos na perspectiva das ciências sociais. História, Ciências, Saúde - Manguinhos 3(3): 485-504.

Freitas N \& Arcuri ASA 1996. Negociação coletiva nacional sobre o benzeno. In M Bonciani (org.) - Saúde, Ambiente e Contrato Coletivo de Trabalho. Ed. LTR, São Paulo.

Garcia R 1986. Los problemas del conocimiento y la perspectiva ambiental del desarrollo Apud: G Duval 1993. Investigación disciplinária y enfoque sistémico. Avance y Perspectiva 12: 67-75.

Howe W, Stonard MD \& Woolen BH 1986. The use of human biological measurements for safety evaluation in the chemical industry. In AN Worden, DV Parke \& J Marks (eds). The Future of Predictive Safety Evaluation, MTP Press, Lancaster.

IARC - International Agency For Research On Cancer 1982. Research on Cancer (IARC) - Chemicals, Industrial Processes and Industries Associated with Cancer in Humans. Monographs on the Evaluation the Carcinogenic Risk of Chemical to Humans. IARC, v.1(suppl.4). Lyon.

IPCS - Programme on Chemical Safety \& IRPTC - International Register of Potentially Toxic Chemicals 1992. Computadorized Listining of Potentially Toxic Chemicals. WHO/UNEP/ILO, Geneva.

Kundiev JI \& Navakatikyan AD 1986. Study of combined effects. In M Karvonen \& MI Mikheev (eds) - Epidemiology of Occupational Health. WHO, Copenhagen, [CWHO European series n.20]. 
Lacaz FAC 1996. Saúde do Trabalhador: um Estudo sobre as Formações Discursivas da Academia, dos Serviços e do Movimento Sindical. Tese de Doutorado. Faculdade de Ciências Médicas, Unicamp, Campinas.

Laurell AC \& Noriega M 1989. Processo de Produção e Saúde: Trabalho e Desgaste Operário. p. 99-144. Hucitec, São Paulo.

Lieber RR 1991. Trabalho em Turnos e Riscos Químicos: O Horário de Trabalho como Fator Interveniente no Efeito Tóxico. Dissertação de Mestrado. Faculdade de Saúde Pública, Universidade de São Paulo São Paulo.

MacClean AEM 1979. Hazards from chemicals: scientific questions and conflicts of interest. Proceedings of the Royal Society of London.8: 179-197.

Monster AC 1988. Biological markers of solvent exposure. Archives of Environmental Health 43(2): 90-91.

MT - Ministério do Trabalho 1993. Secretaria de Segurança e Saúde no Trabalho. Benzeno, D.F. [Subsídios Técnicos].

MT - Ministério do Trabalho 1993. Secretaria de Segurança e Saúde no Trabalho. Portaria n.3, D.O.U. Seção I, n.51, 16/3/94: 3745. D.F.

Novaes TCP 1992. Bases Metodológicas para Abordagem da Exposição ao Benzeno. Dissertação de Mestrado. Instituto de Química, Universidade de São Paulo, São Paulo.

OMS - Organização Mundial da Saúde 1977. Métodos Utilizados para Estabelecer Niveles Admissibles de Exposición Profesional a los Agentes Nocivos. Geneva, [IT 601].

OMS - Organização Mundial da Saúde 1980. Principios $y$ Metodos para Evaluar la Toxicidad de las Substâncias Químicas (I). OPAS/OMS, Mexico, D.C. [Criterios de salud ambiental 6].

OMS - Organização Mundial da Saúde 1981. Efectos sobre la Salud de las Exposiciones Combinadas en el Medio de Trabajo. Genebra [IT 662].

Otway HJ 1985. Regulation and Risk Analysis. p. 1-19. In H Otway \& Peltu M (eds) - Regulating Industrial Risks - Science, Hazards and Public Protection. Butterworths, London.

Porto MFS \& Freitas CM 1997. Análise de riscos tecnológicos ambientais: perspectivas para o campo da saúde do trabalhador. Cadernos de Saúde Pública 13(Supl.2): 109-118.

Renn O 1992. Concepts of risk: a classification, p. 5379. In S Krimsk \& D Golding (eds) - Social Theories of Risk. Praeger, London.

Renn O 1985. Risk analysis: scope and limitations, p. 111-127. In H Otway \& M Peltu (eds) - Regulating Industrial Risks - Science, Hazards and Public Protection. Butterworths, London.
Sass R 1988. What's in a name? The occupational hygienist's problem with threshold limit values. Americal Journal of Industrial Medicine 14: 355-363.

Segurança e Medicina do Trabalho 1985. Portaria 3214/ 78. NR-15. p. 189. 16a ed. Atlas, São Paulo.

Sharlin HI 1989. Risk perception: changing the terms of the debate. Journal of Hazards Mat. 21: 261-272.

Starr C, Rudman R \& Whippe C 1976. Philosophical basis for risk analysis. Anual Review of Energy 1: 629662.

Stengers I 1990. Quem Tem Medo da Ciência? Siciliano, São Paulo.

Susser M 1985. Epidemiology in the United States after World War II: the evolution of technique. Epidemiologic Reviews 7: 147-177.

Tarlau ES 1990. Industrial hygiene with no limits. Guest Editorial. American Industrial. Hygienists Association Journal 51: A-9 e A-10.

Thomas HF 1979. Some observations on occupational higiene standards. American Journal of Industrial Medicine Occupational Hygiene 22: 387-397.

Tyler TR \& Ballantyne B 1988. Pratical assesment and comunication of chemical hazards in the workplace, p. 330-378. In B Ballantyne (ed.) - Perpectives in Basic and Applied Toxicology. Wright, London.

Vanhoorne M 1988. Upgrading occcupational epidemiology and standard setting by improved exposure monitoring. American Journal of Industrial Medicine 14: 733-734.

Verbeek J, Van Dijk FJH \& De Vries FF 1986. Non-auditory effects of noise in industry. International Archives of Environmental Health 58: 333-335.

Vesell ES 1987. Pharmacogenetic perspectives on suscetibility to toxic industrial chemicals. British Journal of Industrial Medicine 44: 505-509.

Waldman EA 1992. Vigilância Epidemiológica como Prática de Saúde Pública. Tese de Doutorado. Faculdade de Saúde Pública, Universidade de São Paulo, São Paulo.

Wünsch Filho V 1992. Riscos Ocupacionais e Câncer de Pulmão. Tese de Doutorado. Faculdade de Saúde Pública, Universidade de São Paulo, São Paulo.

Wynne B 1992. Uncertainty and environmental learning - Reconceiving science and policy in the preventive paradigm. Global Environmental Change 2: 111-127.

Zielhuis RL \& Wibowo AAE 1989. Standard setting in occupational health: philosophical issues. American Journal of Industrial Medicine 16: 569-598. 